\title{
Enfermedad de Kawasaki en lactantes bajo un año de edad. Un reto para el diagnóstico y tratamiento de los pacientes. Experiencia en un centro hospitalario en México
}

\author{
Kawasaki disease in infants under one year of age. A challenge for the diagnosis and treatment of \\ patients. Experience in a hospital center in Mexico
}

Luis Martín Garrido-García1,2, Samia Gaffare-Aranda1, Patricia Cravioto1, Fernando Galván', Rolando Ulloa-Gutierrez ${ }^{2,3}$ y Marco Antonio Yamazaki-Nakashimada1,2

${ }^{1}$ Instituto Nacional de Pediatría. Ciudad de México. México.

${ }^{2}$ Red de Enfermedad de Kawasaki en América Latina (REKAMLATINA).

${ }^{3}$ Hospital Nacional de Niños "Dr. Carlos Sáenz Herrera", San José, Costa Rica.

Financiamiento externo: no hubo.

Los autores declaran la ausencia de conflictos de interés.

Recibido: 15 de julio de 2020 / Aceptado: 12 de septiembre de 2020

\section{Resumen}

Antecedentes: La enfermedad de Kawasaki (EK) en lactantes bajo un año de edad es poco frecuente en la mayoría de los países. Este grupo de pacientes tienen mayor riesgo de desarrollar complicaciones cardiacas. Objetivo: Evaluar el comportamiento clínico, tratamiento empleado y pronóstico cardiaco en lactantes bajo un año de edad atendidos por EK en un hospital pediátrico de tercer nivel en la Ciudad de México. Pacientes y Métodos: Estudio retrospectivo, descriptivo, de pacientes con diagnóstico de EK desde agosto de 1995 a agosto de 2019. Se estudió la presentación clínica, los exámenes de laboratorio, el tratamiento administrado y el desarrollo de lesiones coronarias en pacientes bajo un año de edad y se comparó con pacientes mayores. Resultados: Se estudiaron 687 pacientes, 152 de ellos eran lactantes bajo un año de edad $(22,1 \%)$. Hubo un mayor tiempo al diagnóstico de la EK en los lactantes menores, con un incremento de presentaciones clínicas incompletas; este grupo de pacientes desarrolló en forma más frecuente lesiones coronarias en comparación con los pacientes mayores y también tuvo un mayor porcentaje de aneurismas coronarios gigantes. Hubo dos fallecimientos en los lactantes menores, secundarios a infarto al miocardio. Conclusiones: El diagnóstico de EK en pacientes bajo un año de edad es un reto diagnóstico con presentaciones clínicas incompletas y mayor riesgo de desarrollar complicaciones cardiacas graves.

Palabras clave: enfermedad de Kawasaki; lactantes; arterias coronarias; anomalías; aneurismas.

\begin{abstract}
Background: Frequency of Kawasaki disease (KD) in infants is low in almost all countries. These patients are at higher risk of developing cardiac complications. Aim: To evaluate the clinical features, treatment used and cardiac outcome in infants under one year of age attending for KD in a third level pediatric hospital in Mexico City, Mexico. Methods: A cross-sectional study was conducted in our hospital from August 1995 to August 2019. We analyzed the clinical features, laboratory results, treatment used and cardiac outcomes in infants younger than one year of age and compared them with older patients. Results: We included 687 patients, 152 were younger than one year of age $(22.1 \%)$. There was a delayed diagnosis in younger patients with an increased frequency of incomplete clinical presentations. Coronary artery abnormalities were most common in younger infants who also had an increased frequency of giant coronary artery aneurysms. Two patients in the younger group died in the acute phase of KD of myocardial infarction. Conclusions: Diagnosis of KD in infants younger than 1 year of age is a clinical challenge with an increased rate of incomplete clinical presentations and also an increased risk of development of severe cardiac complications.

Keywords: Kawasaki disease; infants; coronary artery abnormalities; aneurisms.
\end{abstract}


del Corazón (AHA). Se diagnosticó EK completa con la presencia de fiebre elevada de al menos cinco días, más la presencia de cuatro de los cinco criterios clásicos: hiperemia conjuntival bilateral no purulenta, cambios en la mucosa oral, adenopatía cervical mayor de $1,5 \mathrm{~cm}$, exantema morbiliforme y cambios en las extremidades como edema o eritema de palmas y plantas en la fase aguda y descamación en la etapa subaguda. Una forma incompleta se definió con la presencia de fiebre, dos a tres datos clínicos presentes y con la existencia de anomalías coronarias en el ecocardiograma ${ }^{2}$.

Los exámenes de laboratorio analizados fueron hemoglobina, score-z por edad de la hemoglobina, cifra de leucocitos, porcentaje de neutrófilos y de leucocitos inmaduros, plaquetas, velocidad de sedimentación globular, proteína-C reactiva, sodio, albúmina, transaminasas, bilirrubinas y deshidrogenasa láctica.

Para el presente estudio, se revisaron los estudios ecocardiográficos de los pacientes diagnosticados previos al 2011 y se recalculó la definición de lesión coronaria de acuerdo al score-z calculado por peso y talla por la fórmula establecida por Dallaires. Se clasificó como lesión coronaria de acuerdo a los últimos criterios de la AHA, definiéndose una arteria coronaria normal con un score-z menor de 2, dilatación coronaria si el tamaño de la coronaria tenía un score-z entre 2 y 2,5 , aneurisma pequeño con un score-z $>2,5$ a 5,0, aneurisma grande con un score-z $>5,0$ a 10,0 y aneurisma gigante con un score- $\mathrm{z}>10 \mathrm{o}$ un diámetro mayor de $8 \mathrm{~mm}^{2}$.

Los pacientes fueron tratados con IVIG; los primeros pacientes recibieron una dosis de $400 \mathrm{mg} / \mathrm{kg} /$ día durante cinco días y a partir de 1999 todos han sido tratados con IVIG 2 gr/kg en dosis única. Una segunda dosis de IVIG fue administrada en los pacientes con fiebre persistente posterior a $36 \mathrm{~h}$ de la finalización de la IVIG. La IVIG no fue administrada en los pacientes en quienes se diagnosticó la EK en la etapa subaguda de la enfermedad con normalización de los parámetros inflamatorios.

Se usó ácido acetilsalicílico (aspirina) oral, inicialmente a dosis de 80 a $100 \mathrm{mg} / \mathrm{kg} /$ día y, a partir de 2011 , se ha utilizado dosis intermedia (30 y $50 \mathrm{mg} / \mathrm{kg} / \mathrm{día}$ ).

Los corticoesteroides fueron utilizados en aquellos pacientes con riesgo para el desarrollo de complicaciones cardiacas, en aquellos pacientes con disfunción miocárdica o con resistencia a la IVIG.

Las variables continuas se reportaron en media y desviación estándar; la prueba t de Student fue utilizada para analizar las variables continuas y una prueba de $\chi^{2}$ fue utilizada para las variables categóricas. Los valores de $\mathrm{p}<0,05$ fueron considerados como significativos. Se realizó el análisis estadístico con el programa SPSSS versión 21 (SPSS, Chicago, IL, USA).

El presente trabajo fue aprobado por el Comité de Investigación y Ética del Instituto Nacional de Pediatría. 


\section{Resultados}

Durante el período de estudio se diagnosticaron en nuestra institución 754 casos de EK; 67 fueron descartados por no contar con datos completos siendo analizados 687 casos. Del total de pacientes, 152 pacientes eran lactantes menores $(22,1 \%)$; de ellos, 104 fueron varones $(68,4 \%)$.

En los lactantes menores la edad promedio al diagnóstico de EK fue de 8,18 $\pm 2,93$ meses; el paciente más pequeño inició con fiebre a los 15 días de vida y el diagnóstico de la enfermedad se realizó 13 días más tarde. En este grupo, el tiempo desde el inicio de la fiebre hasta

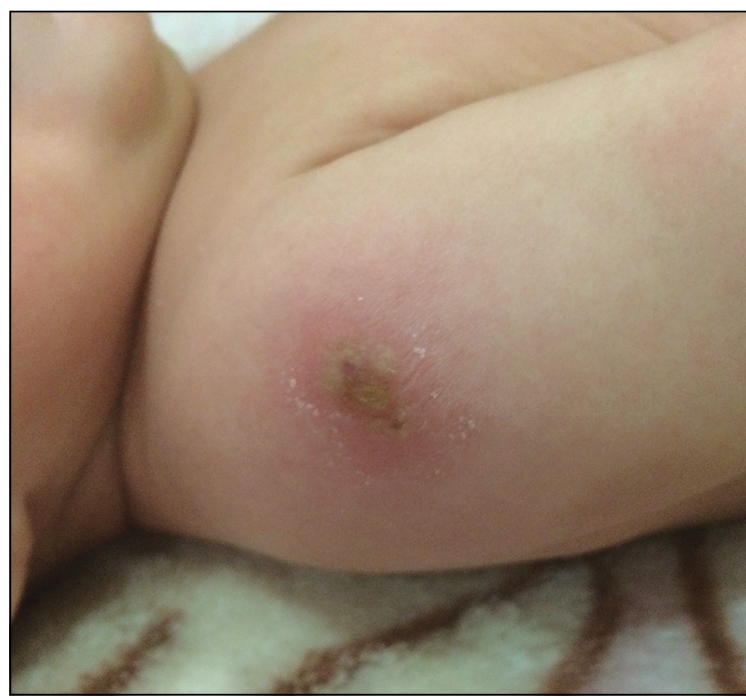

el diagnóstico de la enfermedad fue de 9,65 \pm 6,54 días comparado con 8,59 $\pm 5,33$ días en el grupo de mayor edad $(\mathrm{p}<0,043)$.

De las manifestaciones clínicas clásicas de la EK, la más frecuente en los lactantes menores fueron las alteraciones orales en $89,5 \%$, el exantema se presentó en $85,5 \%$ de los casos, la inyección conjuntival se describió en $84,9 \%$, la hiperemia y/o edema de palmas y plantas en $67,1 \%$ y la adenopatía cervical sólo se presentó en $41,4 \%$ de los casos. Como manifestación no clásica, se encontró un eritema en la cicatriz de BCG en $61,2 \%$ en lactantes menores y sólo en $20,3 \%$ de los pacientes de mayor edad $(\mathrm{p}<0,000)$ (Figura 1). En los lactantes menores, se diagnosticó una forma incompleta de la EK en $29,6 \%$ comparado con $17,8 \%$ en el grupo de mayor edad $(\mathrm{p}<0,001)$.

$\mathrm{Al}$ analizar las formas graves de presentación clínica, los lactantes menores tuvieron choque asociado a la EK en 10 casos $(6,5 \%)$ comparado con 20 casos en el grupo de mayor edad (3,7\%), diferencia sin significancia estadística $(p<0,174)$. Los datos clínicos completos de los lactantes menores y su comparación con el grupo de mayor edad, se muestran en la Tabla 1.

Los lactantes menores tuvieron cifras inferiores de hemoglobina, aunque al realizar la comparación de acuerdo al score-z por edad, no hubo diferencia significativa; por el contrario, en ellos se encontró una mayor cifra de leucocitos, neutrófilos y plaquetas, todos ellos con significancia estadística. Los resultados de los exámenes de laboratorio en lactantes menores y su comparación con pacientes mayores, se muestran en la Tabla 2.
Figura 1. Eritema en el sitio de vacunación de BCG en un paciente de 8 meses de edad con diagnóstico de enfermedad de Kawasaki.

\begin{tabular}{|c|c|c|c|c|c|}
\hline & $\begin{array}{l}\leq 1 \text { año } \\
n=152\end{array}$ & $\%$ & $\begin{array}{l}>1 \text { año } \\
n=535\end{array}$ & $\%$ & $\begin{array}{l}\text { Valor } \\
\text { de } p^{*}\end{array}$ \\
\hline Días al diagnóstico & $9,65 \pm 6,4$ & & $8,59 \pm 5,33$ & & $<0,043$ \\
\hline Sexo masculino & 104 & 68,4 & 347 & 64,9 & 0,498 \\
\hline \multicolumn{6}{|l|}{ Manifestaciones clínicas clásicas } \\
\hline Hiperemia conjuntival & 129 & 84,9 & 487 & 91,0 & $<0,059$ \\
\hline Exantema & 130 & 85,5 & 458 & 85,6 & 1,000 \\
\hline Cambios orales & 136 & 89,5 & 505 & 94,4 & $<0,049$ \\
\hline Cambios en extremidades & 102 & 67,1 & 396 & 74,0 & 0,118 \\
\hline Adenopatía cervical & 63 & 41,4 & 320 & 59,8 & $<0,000$ \\
\hline Eritema en la cicatriz de BCG & 93 & 61,2 & 109 & 20,3 & $<0,000$ \\
\hline Enfermedad de Kawasaki incompleta & 45 & 29,6 & 94 & 17,8 & $<0,001$ \\
\hline Choque asociado a Kawasaki & 10 & 6,6 & 20 & 3,7 & $<0,174$ \\
\hline
\end{tabular}


De los lactantes menores, 138 recibieron tratamiento con IVIG al momento del diagnóstico de la EK (90,1\%); de los 14 que no recibieron IVIG, 71,4\% desarrolló lesiones coronarias comparado con sólo $28,1 \%$ de los pacientes de mayor edad que no recibieron IVIG. La comparación del tratamiento con IVIG y el desarrollo de lesiones coronarias, se muestra en la Tabla 3.

En $10(6,6 \%)$ lactantes menores se indicó una segunda dosis de IVIG por persistencia de la fiebre tras $36 \mathrm{~h}$ de ser administrada, en comparación con 35 pacientes del grupo de pacientes mayores de un año. De los 10 lactantes menores que requirieron una segunda dosis de IVIG, en seis el diagnóstico se realizó antes de los 10 días de inicio de la fiebre. Dentro del grupo de lactantes menores, 75 fueron tratados con corticoesteroides en la etapa aguda (49,3\%). Los lactantes menores con choque (n: 10) recibieron pulsos de metilprednisolona y un paciente requirió el uso de ciclosporina e infliximab por resistencia a IVIG e inflamación persistente.

En el ecocardiograma al diagnóstico de la enfermedad, 19 de los lactantes menores presentaron signos de miocarditis $(12,5 \%)$ comparado con 66 pacientes de los pacientes mayores $(12,3 \%)$. Se encontró efusión pericárdica en 44 pacientes del grupo de menor edad (28,9\%) comparado con 86 casos en los pacientes mayores $(16,0 \%)$ $(\mathrm{p}<0,000)$. Al evaluar las arterias coronarias, 71 de los lactantes menores $(46,7 \%)$ tuvieron algún tipo de lesión coronaria comparado con 133 de los pacientes mayores $(24,8 \%)(\mathrm{p}<0,000)$ y 24 de ellos fueron diagnosticados con la presencia de aneurismas gigantes $(15,8 \%)$ comparado con 19 pacientes en los pacientes mayores $(3,6 \%)$ $(\mathrm{p}<0,000)$.

Fallecieron dos lactantes menores en la etapa aguda $(1,4 \%)$ y ningún paciente en los de mayor edad. En ambos fallecidos se pudo constatar un infarto al miocardio y trombosis de aneurismas gigantes. Un paciente llegó con choque a los cuatro días de iniciada la fiebre y el otro tras 21 días de sintomatología sin diagnóstico.

\section{Discusión}

La EK es considerada, actualmente, la cardiopatía adquirida más común en países desarrollados donde se ha estudiado ampliamente su epidemiología. Desde la creación de REKAMLATINA (Red Latinoamericana para el estudio de la Enfermedad de Kawasaki) en el año 2013, cada vez existen mayores estadísticas de la enfermedad en la región ${ }^{4,6,7}$.

La edad más frecuente de presentación de la EK en la mayoría de los países es alrededor de los 30 meses. $^{8}$ Melonari y cols., en un estudio multicéntrico de la EK en Argentina, encontraron que la edad promedio de presentación fue de $34,8 \pm 26,2$ meses y sólo $16,6 \%$ de los

\begin{tabular}{|c|c|c|c|}
\hline & $\begin{array}{l}\leq 1 \text { año } \\
\mathrm{n}=152\end{array}$ & $\begin{array}{l}>1 \text { año } \\
n=535\end{array}$ & $\begin{array}{c}\text { Valor de } \\
p^{*}\end{array}$ \\
\hline Hemoglobina (g/dl) & $10,80 \pm 1,51$ & $11,84 \pm 1,61$ & $<0,000$ \\
\hline z-score hemoglobina & $-1,19 \pm 1,51$ & $-1,57 \pm 1,61$ & 0,454 \\
\hline Leucocitos $\left(\mathrm{mm}^{3}\right)$ & $16.630 \pm 6.881$ & $13.440 \pm 6.493$ & $<0,000$ \\
\hline Neutrófilos (\%) & $61,33 \pm 19,21$ & $70,73 \pm 18,89$ & $<0,001$ \\
\hline Plaquetas $\left(\mathrm{mm}^{3}\right)$ & $464.818 \pm 224.132$ & $372.781 \pm 203.685$ & $<0,000$ \\
\hline VSG $(\mathrm{mm} / \mathrm{h})$ & $44,19 \pm 15,32$ & $43,18 \pm 15,36$ & 0,507 \\
\hline PCR (mg/dl) & $8,07 \pm 6,79$ & $9,95 \pm 17,96$ & 0,291 \\
\hline Albúmina (mg/dl) & $3,06 \pm 0,58$ & $3,15 \pm 1,79$ & 0,507 \\
\hline Sodio (mEq/lt) & $136,2 \pm 3,5$ & $134,9 \pm 10,3$ & 0,578 \\
\hline AST (IU/It) & $54,62 \pm 44,35$ & $70,28 \pm 84,52$ & $<0,041$ \\
\hline ALT (IU/lt) & $60,52 \pm 81,17$ & $75,48 \pm 87,50$ & $<0,079$ \\
\hline Bilirrubina total (mg/dl) & $0,78 \pm 0,85$ & $1,93 \pm 1,49$ & 0,415 \\
\hline Bilirrubina directa (mg/dl) & $0,29 \pm 0,57$ & $0,55 \pm 1,00$ & $<0,011$ \\
\hline Bilirrubina indirecta $(\mathrm{mg} / \mathrm{dl})$ & $0,46 \pm 0,37$ & $0,65 \pm 0,80$ & $<0,016$ \\
\hline DHL (IU/It) & $288,51 \pm 93,64$ & $303,76 \pm 284,01$ & 0,554 \\
\hline \multicolumn{4}{|c|}{$\begin{array}{l}\text { VSG }=\text { velocidad de sedimentación globular, } \mathrm{PCR}=\text { proteína } \mathrm{C} \text {-reactiva, } \mathrm{AST}=\text { aspartato amin } \\
\text { transferasa, } \mathrm{ALT}=\text { alanino amino transferasa } \mathrm{DHL}=\text { deshidrogenasa láctica. }{ }^{*} \text { Valor de } \mathrm{p}<0,0 \\
\text { significancia estadística. }\end{array}$} \\
\hline
\end{tabular}

Tabla 3. Comparación del tratamiento y lesiones cardiacas de los pacientes con enfermedad de Kawasaki de acuerdo a la edad de presentación $(n=687)$

\begin{tabular}{|c|c|c|c|c|c|}
\hline & $\begin{array}{l}\leq 1 \text { año } \\
n=152\end{array}$ & $\%$ & $\begin{array}{l}>1 \text { año } \\
n=535\end{array}$ & $\%$ & $\begin{array}{c}\text { Valor de } \\
p^{*}\end{array}$ \\
\hline \multicolumn{6}{|l|}{ Tratamiento } \\
\hline No IVIG & 14 & 9,2 & 32 & 3,5 & \\
\hline Días al diagnóstico sin IVIG & \multicolumn{2}{|c|}{$16,17 \pm 7,65$} & \multicolumn{2}{|c|}{$15,22 \pm 6,53$} & $<0,052$ \\
\hline Reciben IVIG & 138 & 90,1 & 497 & 92,3 & 0,466 \\
\hline Días al diagnóstico con IVIG & \multicolumn{2}{|c|}{$9,10 \pm 6,27$} & \multicolumn{2}{|c|}{$8,13 \pm 4.85$} & 0,678 \\
\hline Segunda dosis IVIG & 10 & 6,6 & 35 & 6,5 & 1,000 \\
\hline Corticoesteroides & 75 & 49,3 & 247 & 46,1 & 0,398 \\
\hline \multicolumn{6}{|l|}{ Ecocardiograma al diagnóstico } \\
\hline Miocarditis & 19 & 12,5 & 66 & 12,3 & 0,887 \\
\hline Efusión pericárdica & 44 & 28,9 & 86 & 16,0 & $<0,000$ \\
\hline \multicolumn{6}{|l|}{ Lesiones coronarias } \\
\hline Aneurismas coronarios global & 71 & 46,7 & 133 & 24,8 & $<0,000$ \\
\hline Aneurismas coronarios sin IVIG & 10 & 71,4 & 9 & 28,1 & $<0,024$ \\
\hline Aneurismas coronarios con IVIG & 64 & 46,4 & 124 & 24,7 & $<0,000$ \\
\hline Aneurismas gigantes global & 24 & 15,8 & 19 & 3,6 & $<0,000$ \\
\hline Aneurismas gigantes sin IVIG & 3 & 21,4 & 0 & 0 & $<0,014$ \\
\hline Aneurismas gigantes con IVIG & 21 & 15,2 & 19 & 3,8 & $<0,000$ \\
\hline
\end{tabular}


casos estudiados fueron en lactantes menores ${ }^{9}$. Resultados similares fueron reportados en España (KAWA-RACE) con una mediana de edad al diagnóstico de 33,6 meses, y siendo sólo $16,5 \%$ de ellos lactantes menores ${ }^{10}$. Borzutzky y cols, en un estudio de siete años de la EK en Chile, reportaron que $25 \%$ de los 786 casos estudiados eran lactantes menores ${ }^{11}$.

Se ha reportado que existen diferencias clínicas entre las diferentes edades de presentación de la $\mathrm{EK}^{12,13}$. En el presente estudio, las manifestaciones clínicas clásicas de la EK menos frecuentes fueron las lesiones en las extremidades y la adenopatía cervical unilateral. De acuerdo a lo reportado por otros autores, la adenopatía cervical unilateral es el signo clásico de la EK menos frecuente en pacientes de menor edad ${ }^{14-16}$.

Otro aspecto muy importante a considerar en el cuadro clínico de la EK en menores de edad, es un incremento en las formas incompletas de la enfermedad en comparación con pacientes de mayor edad ${ }^{17}$. En nuestro estudio encontramos que los lactantes menores tuvieron un incremento estadísticamente significativo de EK incompleta. Este hallazgo es muy parecido a lo reportado por Budnik y cols., en una serie clínica chilena en que de los pacientes bajo 2 años de edad con EK, 27,3\% tuvo una forma incompleta de la enfermedad ${ }^{18}$. Estos resultados, son muy semejantes al estudio nacional español donde el porcentaje de presentación incompleta en lactantes menores fue de $25 \%{ }^{10}$. Sin embargo, esta cifra se incrementó hasta $51 \%$ de los pacientes estudiados por Salgado y cols., en el área de San Diego, Estados Unidos de América ${ }^{14}$. En un estudio reciente de REKAMLATINA donde se analizó el comportamiento clínico de los pacientes bajo 6 meses de edad con EK, se encontró que 55,6\% de los pacientes tuvo una forma incompleta de la enfermedad ${ }^{4}$. En Corea, Yeo y cols., también encontraron que hubo una presentación incompleta en 56,6\% de los lactantes menores estudia$\operatorname{dos}^{19}$. Esta cifra se incrementó hasta $68,7 \%$ de los casos bajo un año de edad, en un estudio multicéntrico italiano ${ }^{20}$.

Las presentaciones incompletas de la EK en pacientes de menor edad, pueden favorecer el retardo en el diagnóstico. En el presente estudio, en los lactantes menores, el diagnóstico de la EK se realizó más tardíamente, lo cual tiene implicaciones en el desarrollo de complicaciones cardiacas. En un estudio previo en nuestro hospital, realizado por Garrido-García y cols., encontramos que el diagnóstico de la EK se realiza cada vez en forma más temprana $^{21}$. Singh y cols., reportaron que el diagnóstico de EK se tardó por más de 10 días en $70 \%$ de los lactantes menores estudiados ${ }^{22}$. En países con mayor de incidencia de EK como Japón y Corea, el diagnóstico de la enfermedad se hace en forma más temprana (cercano a los 6 días), pero también fue un poco más prolongado en los pacientes de menor edad ${ }^{12,13}$.

El eritema en el sitio de la cicatriz de la vacuna de BCG es un dato clínico muy importante para el diagnóstico de la EK en pacientes de menor edad en países donde se administra la vacuna de BCG como parte del esquema de vacunación, tales como México. En otra publicación, Garrido-García y cols., describieron que el eritema en el sitio de la cicatriz de BCG se presentó en 32,3\% de los lactantes menores ${ }^{23}$. Este hallazgo también ha sido reportado en Japón, donde se encontró que el eritema en el sitio de la vacuna de BCG (70\%) fue más frecuente en pacientes con EK bajo 20 meses de edad que la presencia de linfadenopatía cervical ${ }^{24}$. Es importante señalar que el eritema de la cicatriz de la vacuna de BCG no sólo ha sido utilizado para ayudar al diagnóstico de la EK, sino también para hacer el diagnóstico temprano de la enfermedad en los lactantes menores con EK, ya que se ha descrito que este eritema se presenta en los primeros días de aparición de la fiebre ${ }^{25,26}$.

Un aspecto muy importante que refleja la gravedad de la EK en pacientes de menor edad fue la manifestación de choque al ingreso. Esto también ha fue reportado en la región por Ulloa-Gutierrez y cols., al analizar choque asociado a la EK y recientemente por Moreno y cols., al describir el comportamiento clínico de lactantes bajo 6 meses de edad en América Latina ${ }^{4,27}$.

En cuanto a los exámenes de laboratorio, no encontramos diferencia significativa en la concentración de hemoglobina entre lactantes menores y los pacientes mayores; sí la hubo al comparar el conteo de leucocitos, porcentaje de neutrófilos, conteo de plaquetas y enzimas hepáticas siendo significativamente mayores los recuentos de leucocitos y plaquetas en lactantes menores. Estos datos pueden reflejar una mayor inflamación en los pacientes con EK de menor edad, que tiene implicaciones en el desarrollo de lesiones coronarias ${ }^{14,15,28}$.

El aspecto clínico más importante en los lactantes menores con EK es que tienen una enfermedad más grave y un riesgo incrementado de afectación cardiaca. En nuestro estudio, encontramos que los lactantes menores tuvieron mayor incidencia de efusión pericárdica al momento del diagnóstico. Esta alteración pericárdica es más común en pacientes con $\mathrm{EK}$ que desarrollan lesiones coronarias y es un hallazgo cardiaco que refleja una mayor inflamación. También encontramos una mayor frecuencia de lesiones coronarias en nuestro grupo de pacientes de menor edad. Esta respuesta ha sido encontrada por prácticamente todos los autores que han estudiado lactantes menores con $\mathrm{EK}^{12,20}$. El incremento en la incidencia de lesiones coronarias en lactantes menores con EK también fue encontrada en un estudio de cinco años en niños españoles con EK (20,0 vs $9,6 \%)(p<0,001)^{10}$. Cameron y cols., estudiaron el comportamiento de lactantes menores con EK en Chicago y reportaron que este grupo de pacientes no sólo tenía un riesgo incrementado en relación a pacientes mayores 
más de cinco días de duración, incluso en ausencia de los signos clásicos de la enfermedad ${ }^{2}$.

Este estudio tiene algunas limitaciones; la primera de ellas es su carácter retrospectivo y, por ende, algunas de las variables recientemente estudiadas en los pacientes, no pudieron ser conocidas en nuestros primeros pacientes. En segundo lugar, los datos provienen de un solo centro hospitalario con una muestra pequeña comparada con otros estudios epidemiológicos de países con mayor incidencia de EK como Japón. Sin embargo, el Instituto Nacional de Pediatría es uno de los hospitales pediátricos de mayor referencia de niños con EK y hasta nuestro conocimiento es el estudio con mayor número de lactantes menores con EK en América Latina.

\section{Conclusiones}

El diagnóstico temprano y tratamiento oportuno de los lactantes menores con diagnóstico de EK es un reto ya que la mayoría de este grupo de pacientes tiene una mayor frecuencia de formas incompletas de la enfermedad, lo que hace difícil reconocerla y establecer un tratamiento adecuado. La reactivación de la vacuna de BCG en países donde es parte del esquema de vacunación puede ayudar a lograr un diagnóstico temprano de la EK. Este grupo de pacientes tiene un riesgo incrementado de desarrollar complicaciones cardiacas graves.

\section{Referencias bibliográficas}

1.- Newburger J W, Takahashi M, Burns J C. Kawasaki disease. JACC 2016; 67: 1738-49. doi: 10.1016/j.jacc.2015.12.073.

2.- McCrindle B W, Rowley A H, Newburger J W, Burns J C, Bolger A F, Gewitz M, et al. Diagnosis, treatment, and long-term management of Kawasaki disease: a statement for health professionals from American Heart Association. Circulation 2017; 135:e927-e999. doi: 10.1161/CIR.0000000000000484.

3.- Yim D, Curtis N, Cheung M, Burgner D. An update on Kawasaki disease II: clinical features, diagnosis, treatment and outcomes. J Paediatr Child Health 2013; 49: 614-23. doi: 10.1111/jpc.12221.

4.- Moreno E, García S D, Bainto E, Salgado A P, Parish A, Rosellini B D, et al. Presentation and outcomes of a Kawasaki disease in Latin American infants younger than 6 months of age: A multinational multicenter study of the REKAMLATINA network. Front Pediatr 2020; 8: article 384. doi: 10.3389/fped.2020.00384.

5.- Dallaire F, Dahdah N. New equations and a critical appraisal of coronary artery z-scores in healthy children. J Am Soc Echocardiogr 2011; 24: 60-74. doi: 10.1016/j.echo.2010.10.004.
6.- Ulloa-Gutierrez R, Salgado A P, Tremoulet A H Kawasaki disease in Latin-American children: past, current and future challenges. J Pediatr Infect Soc 2014; 3: 280-1. doi: 10.1093/jpids/ piu 105.

7.- Garrido-García L M, Gutierrez-Alanis J H, Ramírez-Perea A I, Tremoulet A, YamazakiNakshimada M A. Kawasaki disease in infants in the first 3 months of age in a Mexican population. Front Pediatr 2020; 8: 397. doi:10.3389/fped.2020.00397.

8.- Uehara R, Belay E D, Epidemiology of Kawasaki disease in Asia, Europe, and the United States. J Epidemiol. 2012; 22: 79-85. doi: 10.2188/jea.je20110131.

9.- Melonari P, Abate H, Llano-López L H, Cutica R J, Apaza T, Battagliotti C, et al. Características clinico-epidemiológicas y predictores de complicaciones coronarias en niños de Argentina con enfermedad de Kawasaki. Rev Chilena Infectol 2019; 36: 63641. doi: 10.4067/S0716-10182019000500636.

10.- Fernández-Cooke E, Barrios-Tascón A, Sánchez-Manubens J, Antón J, Grasa-Lozano C D, Aracil-Santos J, et al. Epidemiological and clinical features of Kawasaki disease in Spain over 5 years and risk factors for aneurysm development. (2011-2016). KAWA-RACE study group. PLoS One 2019;14 (5): e0215665. Published 2019 May 20. doi:10.1371/journal. pone.0215665.

11.- Borzutzky A, Hoyos-Bachiloglu R, Cerda J, Talesnik E. Rising hospitalizations rates of Kawasaki disease in Chile between 2001 and 2007. Rheumatol Int 2012; 32: 22491-5. doi:10.1007/s00296-011-2050-4.

12.- Shiozawa Y, Inuzuka R, Harita Y, Kagawa J. Age-related differences in the course of the acute phase symptoms of Kawasaki disease. Ped Infect Dis J 2013; 32:e365-e369. doi: 10.1097/INF.0b013e3182952027.

13.- Lee K Y, Hong J H, Han J W, Lee J S, Lee B C, Burgner D. Features of Kawasaki disease in the extremes of age. J Paediatr Child Health 2006; 42: 423-7. doi: 10.1111/j.14401754.2006.00898.x.

14.- Salgado A P, Ashouri N, Berry E K, Sun X, Jain S, Burns J C, et al. High risk of coronary artery aneurysms in infants younger than 6 months of age with Kawasaki disease. J Pediatr 2017; 185: 112-6. doi: 10.1016/j. jpeds.2017.03.025.

15.- Shi L, Li J, Qie D, Hua X, Pan J, Shi X, et al. Clinical manifestations of Kawasaki disease in different age groups: retrospective data from southwest China. [published online ahead of 
print, 2020 May 4]. Clin Rheumatol 2020; 10.1007/s10067-020-05069-5. doi: 10.1007/ s10067-020-05069-5.

16.- Liu J C, Lo C W, Hwang B, Lee P C. Clinical manifestations vary with different age spectrums in infants with Kawasaki disease. Scie World J 2012; article ID: 210382. doi:10.1100/2012/210382.

17.- No S J, Kim D O, Choi K M, Eun L Y. Do predictors of incomplete Kawasaki disease exist for infants? Pediatr Cardiol 2013; 34. 286-90. doi: 10.1007/s00246-012-0440-3.

18.- Budnik I, Hirsch T, Fernández C, Yáñez L, Zamorano J. Enfermedad de Kawasaki: una serie clínica. Rev Chilena Infectol 2011; 28: 416-22. http://dx.doi.org/10.4067/S071610182011000600005

19.- Yeo Y, Kim T Y, Ha K S, Jang G Y, Lee J H, Lee $\mathrm{K} \mathrm{C}$, et al. Incomplete Kawasaki disease in patients younger than 1 year of age: a possible inherent risk factor. Eur J Pediatr 2009; 168: 157-62. doi: 10.1007/s00431-008-0722.

20.- Mastrangelo G, Cimaz R, Battista-Calabri G, Simonini G, Lasagni D, Resti M, et al. Kawasaki disease in infants less than one year of age: an Italian cohort from a single center. BMC Pediatr 2019; 19: article: 321. doi: 10.1186/s12887-019-1695-0.

21.- Garrido-García L M, Soto-Blanquel J L, Espinosa-Rosales F J. Enfermedad de Kawasaki: Cuadro clínico, exámenes de laboratorio y lesiones coronarias. Acta Ped Mex 2015; 36: 314-21. http://www.scielo.org.mx/ $\mathrm{pdf} / \mathrm{apm} / \mathrm{v} 36 \mathrm{n} 4 / \mathrm{v} 36 \mathrm{n} 4 \mathrm{a} 2 . \mathrm{pdf}$

22.- Singh S, Agarwal S, Bhattad S, Gupta A, Suri D, Rawat A, et al. Kawasaki disease in infants below 6 months: a clinical conundrum? Int J Rheum Dis 2016; 19: 924-8. doi: 10.1111/1756$185 X .12854$.

23.- Garrido-García L M, Castillo-Moguel A, Vázquez-Rivera M, Cravioto P, Fernando G. Reaction of the BCG scar in the acute phase of
Kawasaki disease in Mexican children. Pediatr Infect Dis J 2017; 36: 3237-e241. doi: 10.1097/ INF.0000000000001633.

24.- Uehara R, Igarashi H, Yashiro M, Nakamura Y, Yanagawa H. Kawasaki disease patients with redness or crust formation at the Bacille Calmette-Guérin inoculation site. Pediatr Infect Dis J 2010; 29: 430-3. doi: 10.1097/ INF.0b013e3181 cacede.

25.- Kang J H, Hong S J, Seo I A, Kwak M H, Cho S M, Kim D W, et al. Early detection of Kawasaki disease in infants. Korean Circ J 2015; 45: 510-5. doi: 10.4070/ kcj.2015.45.6.510.

26.- Chacón-Cruz E, Garrido-García L M, Saltigeral-Simental P, Faugier-Fuentes E, Yamazaki-Nakashimada M A, MartínezMedina L, et al. Time of appearance and type of BCG scar reactions in children with Kawasaki disease: A 3-year prospective multicenter, descriptive study at 17 Mexican referral hospitals. En $36^{\text {th }}$ Annual Meeting of the European Society for Paediatric Infectious Diseases (ESPID 2018). Malmö, Sweden. May $28^{\text {th }}$, 2018. E-poster ESP18-0949 \& poster discussion rounds.

27.- Ulloa-Gutierrez R, Faugier-Fuentes E, Garrido-García L M, Luque M T, Collia A, Chacón-Cruz E, et al. Kawasaki Disease Shock Syndrome in children from 20 Latin American countries: A 3-year prospective multinational, multicenter analysis of the REKAMLATINA Network (period June 2014-May 2017). En $12^{\text {th }}$ International Kawasaki Disease Symposium. Yokohama, Japan June 12-15, 2018. Poster 186.

28.- Yoon Y M, Yun H W, Kim S H. Clinical characteristics of Kawasaki disease in infants younger than 6 months: A single-center study. Korean Circ J 2016; 46: 550-5. doi: 10.4070/ kcj.2016.46.4.550.

29.- Cameron S A, Carr M, Pahl E, DeMaris N, Shulman S T, Rowley A H. Coronary artery aneurysms are more severe in infants than in older children with Kawasaki disease. Arch Dis Child 2019:104: 451-5. doi 10.1136/ archdischild-2018-314967.

30.- Garrido-García L M, Moran-Villaseñor E, Yamazaki-Nakashimada M A, Cravioto P, Galván F. Giant coronary artery aneurysms complicating Kawasaki disease in Mexican children. Cardiol Young 2017; 28: 386-90. doi: $10.1017 / \mathrm{S} 1047951117001470$.

31.- Bayers S, Shulman S T, Paller A S. Kawasaki disease. Part II. Complications and treatment. J Am Acad Dermatol 2013; 69: 513-22. doi: 10.1016/j.jaad.2013.06.040.

32.- Kobayashi T, Inoue Y, Takeuchi K, Okada Y, Tamaura K, Tomomasa T, et al. Prediction of intravenous immunoglobulin unresponsiveness in patients with Kawasaki disease. Circulation 2006; 113: 2606-12. doi: 10.1161/ CIRCULATIONAHA.105.592865.

33.- Egami K, Muta H, Ishii M, Suda K, Sugahara Y, Lemura M, et al. Predition of resistance to intravenous immunoglobulin treatment in patients with Kawasaki disease. J Pediatr 2006; 149: 237-40. doi: 10.1016/j. jpeds.2006.03.050.

34.- Wu S, Long Y, Chen S, Huang Y, Liao Y, Sun $\mathrm{Y}$, et al. A new scoring system for prediction of intravenous immunoglobulin resistance of Kawasaki disease in infants under 1-year old. Front Pediatr 2019; 7: article 514.. doi:10.3389/ fped.2019.00514.

35.- Ulloa-Gutierrez R, Saltigeral-Simental P, Faugier-Fuentes E, Yamazaki-Nakashimada M A, Martínez-Medina L, Gámez-González L B, et al. Estudio prospectivo, descriptivo multicéntrico de la Red REKAMLATINA sobre la enfermedad de Kawasaki (EK) en infantes < 6 meses en 17 hospitales de México: junio 2014-mayo 2017. En XX Congreso Interamericano de Pediatría. Ciudad de México. México enero 30, 2018. Poster 61 . 